$\underline{\text { Hematology }}$

\title{
Evaluation of Vaso-occlusive Crises in United States Sickle Cell Disease Patients: A Retrospective Claims-based Study
}

\author{
Nirmish Shah ${ }^{1}$, Menaka Bhor ${ }^{2}$, Lin Xie ${ }^{3,4}$, Rashid Halloway ${ }^{2}$, Steve Arcona ${ }^{2}$, Jincy Paulose ${ }^{2}$, Huseyin Yuce \\ ${ }^{1}$ Duke University, Durham, NC, USA \\ ${ }^{2}$ Novartis Pharmaceuticals Corporation, East Hanover, NJ \\ ${ }^{3}$ SIMR, Inc., Ann Arbor, MI \\ ${ }^{4}$ New York City College of Technology, CUNY, New York, NY \\ *Corresponding author: menaka.bhor@novartis.com
}

\begin{abstract}
Sickle cell disease (SCD) is a life-threatening vascular disease that burdens affected persons physically. SCDrelated vaso-occlusive crises (VOCs) are one of the primary causes of morbidity and mortality. Our objective was to examine the epidemiology of pain crises and the relationship between pain crises and major acute complications among SCD patients. Using the Medicaid Analytic Extracts from 2009-2013, patients with SCD were selected and the first clinical claim indicating SCD during the identification period was defined as the index date. Patients were required to have continuous Medicaid enrollment for $\geq 6$ months pre- and 12 months postindex period. Clinical outcomes included mortality, inpatient pain crises, and complications. Cox regressions were applied to examine the relationship between pain crises and deaths or acute complications, respectively. A total of 20909 patients were included with a mean age of 17.9 years. The rate of VOC events in 100 person-years was 142.20 for adults and 53.91 for pediatric patients. Patients with VOCs were associated with a higher risk for death (hazard ratio $=1.56$; $95 \%$ confidence interval: [1.19-2.05]) or acute complications including acute chest syndrome, stroke, pulmonary embolism, splenic sequestration, and pulmonary hypertension. SCD patients have a substantial burden of disease-related complications. This study suggests that inpatient vasoocclusive crisis is a key risk factor for acute complications.
\end{abstract}

Keywords: red cells, iron, erythropoiesis 


\section{Background}

Sickle cell disease (SCD) is a life-threatening vascular disorder, which early on progresses into a systemic disease. ${ }^{1,2}$ SCD can affect various races and occurs more often among African-American and Hispanic-American births. ${ }^{3}$ SCD affects $\sim 100000$ Americans. $^{4}$

Vaso-occlusive crises (VOCs), also known as acute sickle cell pain crises, are the most common, painful complication of the disease and the main reason why patients seek medical care in hospitals. Vaso-occlusive events are driven by endothelium damage/dysfunction and inflammation, which can then lead to vascular damage. SCD patients' erythrocytes have the propensity to change into crescent shapes with abnormal adhesive properties, which increase interactions with white blood cells, platelets, endothelial cells, and extracellular matrix proteins. All these abnormal interactions accelerate the development of clinical vasculopathy and promote cerebrovascular and pulmonary vascular diseases. VOC significantly affects patients' quality of life and often requires emergency care. ${ }^{5}$ Several diary studies of SCD patients have reported high rates of pain as well as an intense severity of pain during study days ${ }^{6-8} \mathrm{VOC}$ is the leading cause of hospitalization among SCD patients, ${ }^{9}$ and health care utilization (HRU) among SCD patients remains high due to patients' difficulty with acute pain management and the lack of new effective therapies. ${ }^{10}$

SCD-related complications are associated with early mortality and high morbidity, and VOC is often a precursor of these complications. ${ }^{11}$ In the 1990s, the majority of deaths among SCD patients occurred prior to the third year of life and mainly due to infections, acute chest syndrome (ACS), and splenic sequestration crises. ${ }^{12}$ Vaccination advancements have largely decreased early childhood mortality among SCD patients, but life expectancy has not improved much for adult SCD patients over the last era. ${ }^{13}$ While death among SCD patients is often unexpected, and the direct cause of death unclear, cardiopulmonary diseases including ACS, stroke, and pulmonary hypertension remain the most commonly reported complications associated with death. ${ }^{14,15}$ Due to the common mechanism of vaso-occlusion and SCD-related complications, VOC events have been found to precede other acute complications such as ACS in up to $80 \%$ of cases. ${ }^{16}$ Most patients initially seek acute care for a VOC episode; however, as the crisis event is now recognized as a primary risk factor for additional life-threatening complications, ${ }^{11} \mathrm{VOC}$ has become a marker of disease severity and an indicator of premature mortality in the modern patient cohort. ${ }^{17}$

Though pediatric and adult SCD patients experience many similar symptoms and management issues, SCD complications and disease outcomes usually differ by age. ${ }^{18}$ Hydroxyurea has improved health outcomes in both populations. Health outcomes have improved and stabilized in the pediatric population, but the adult population has experienced an increasing mortality rate: there is a great unmet need for new therapies and an understanding of the underlying disease factors contributing to the increased mortality. Further, hydroxyurea will not fully resolve the symptoms of a sizeable proportion of patients in both age cohorts, leading to an unmet need in both populations. ${ }^{19}$ To understand the burden and impact of VOC among SCD patients in a real-world setting, this study evaluated the rate of complications and associations between VOC and lifethreatening complications.

\section{Methods}

\section{Data Source}

This was a retrospective, observational cohort study using US Medicaid databases. The study period ranged from January 1 1s, 2009 through December 31 $1^{\text {st }}, 2013$, with the identification period between July $1^{\text {st }}, 2009$ and December $31^{\text {st }}, 2012$ so that every patient would have a $\geq 6$-month baseline and $\geq 1$-year follow-up period. 
The Medicaid Analytic Extracts (MAX) data system contains extensive individual-level information on the characteristics of Medicaid enrollees in all 50 states and the District of Columbia as well as the services used during a calendar year. Specifically, MAX consists of one personal summary file and four claims files that provide fee-for-service claims, managed care encounter data, and premium payments. The study included feefor-service patients from all available states and Managed Care enrollees who resided in 14 states with relatively complete data: Arizona, California, Indiana, Kansas, Kentucky, Minnesota, Nebraska, New Jersey, New Mexico, New York, Oregon, Tennessee, Texas, and Virginia. Service use among Managed Care enrollees is captured in encounter data. Medicaid enrollees who had dual edibility with Medicare are not included in this study due to incomplete information in the MAX data.

\section{Patient Selection}

Patients were included in the study if they had $\geq 1$ clinical claim code related to SCD (International Classification of Diseases, $9^{\text {th }}$ Revision, Clinical Modification [ICD-9-CM] codes 282.41, 282.42, 282.60-282.69) during the identification period (July $1^{\text {st }}, 2009$ to December 31 ${ }^{\text {st }}$, 2012). Patients were required to have continuous health plan enrollment with medical and pharmacy benefits during the 6 months before the index date (baseline period) and 1 year after the index date and to not be enrolled in a clinical trial during the study period (identified using ICD-9-CM code V70.7). The first observed SCD-related clinical code during the identification period was designated as the index date. Patient data were assessed until the earliest of disenrollment, death, or the end of the study period.

\section{Baseline Measures}

Variables during the 6-month baseline period were measured. Sociodemographic variables including age, sex, race, and US geographic region (Northeast, North Central, South, and West) were flagged during the patient selection process. The baseline Charlson comorbidity index (CCI) score was calculated based on the most updated version of the CCI. ${ }^{20}$ Baseline individual comorbid conditions were flagged including VOC, pulmonary conditions such as ACS, cerebrovascular conditions (stroke), hepatic conditions (gallstones), splenic conditions (splenic sequestration), and other conditions that commonly occurred among SCD patients. Medications and management procedures frequently used for the SCD population were identified during the baseline period. Baseline all-cause HRU were also identified by inpatient, outpatient (emergency room, office, other), and pharmacy visits.

\section{Outcome Measures}

Vaso-occlusive events were defined as an inpatient stay with a primary or secondary clinical claim of SCD with crisis (ICD-9-CM: 282.42, 282.62, 282.64, 282.69) within the SCD population. The event rate of VOC episodes after the index SCD diagnosis was calculated in 100 person-years using the number of events divided by the length of the follow-up period. Deaths were identified by flagging patients who died during the entire follow-up period. The rate of complications was identified for cerebrovascular, hepatic, pulmonary, and splenic conditions. All the clinical events were evaluated separately for pediatric patients (aged $<18$ ) and adult patients (aged $\geq 18$ ). Life-threatening complications including ACS, splenic sequestration, pulmonary embolism, stroke, and pulmonary hypertension were identified by ICD-9-CM diagnosis code.

\section{Statistical Methods}

All variables were first analyzed descriptively. Percentages and numbers were provided for categorical variables. Means and standard deviations were provided for continuous variables. 
Cox proportional hazards regression was used for the multivariate analysis of the time to first complication after the index date, concerning the relationship between the rate of follow-up VOC and life-threatening complications requiring acute care-including ACS, splenic sequestration, pulmonary embolism, stroke, pulmonary hypertension, and death. For the Cox model, the rate of follow-up VOC events before the complication and death were identified. Stepwise model selection was applied. Considering the possibility of progression of diseases with time, follow-up VOCs were controlled in the model as time-varying variables. The impact of the complications was also controlled in the model. For example, if stroke is the dependent outcome, then other complications (i.e., ACS, splenic sequestration, pulmonary embolism, pulmonary hypertension) were put in the model. All complications were controlled in the model for the death outcome. Patient demographics and baseline clinical characteristics including age, sex, race, geographic region, CCI scores, baseline SCD management including SCD medication use and SCD management procedures (e.g., blood transfusion and Transcranial Doppler Ultrasonography), and baseline HRU; the number of baseline VOC events were controlled in the model as covariates. Hazard ratios (HR), 95\% confidence intervals (CI), and p-values were examined for the follow-up VOC and all other covariates.

\section{Data Sharing Statement}

The data analyzed during the current study are available from the corresponding author on reasonable request.

\section{Results}

\section{Baseline Characteristics for Overall SCD Patients}

After applying the inclusion and exclusion criteria, a total of 20909 SCD patients who met the study selection criteria were included (Figure 1).

\section{Figure 1. Patient Selection Flow Chart}

$\geq 1$ diagnosis claim with SCD during the identification period (01JUL2009-

31DEC2012). The first observed SCD diagnosis during the identification period was designated as the index date.

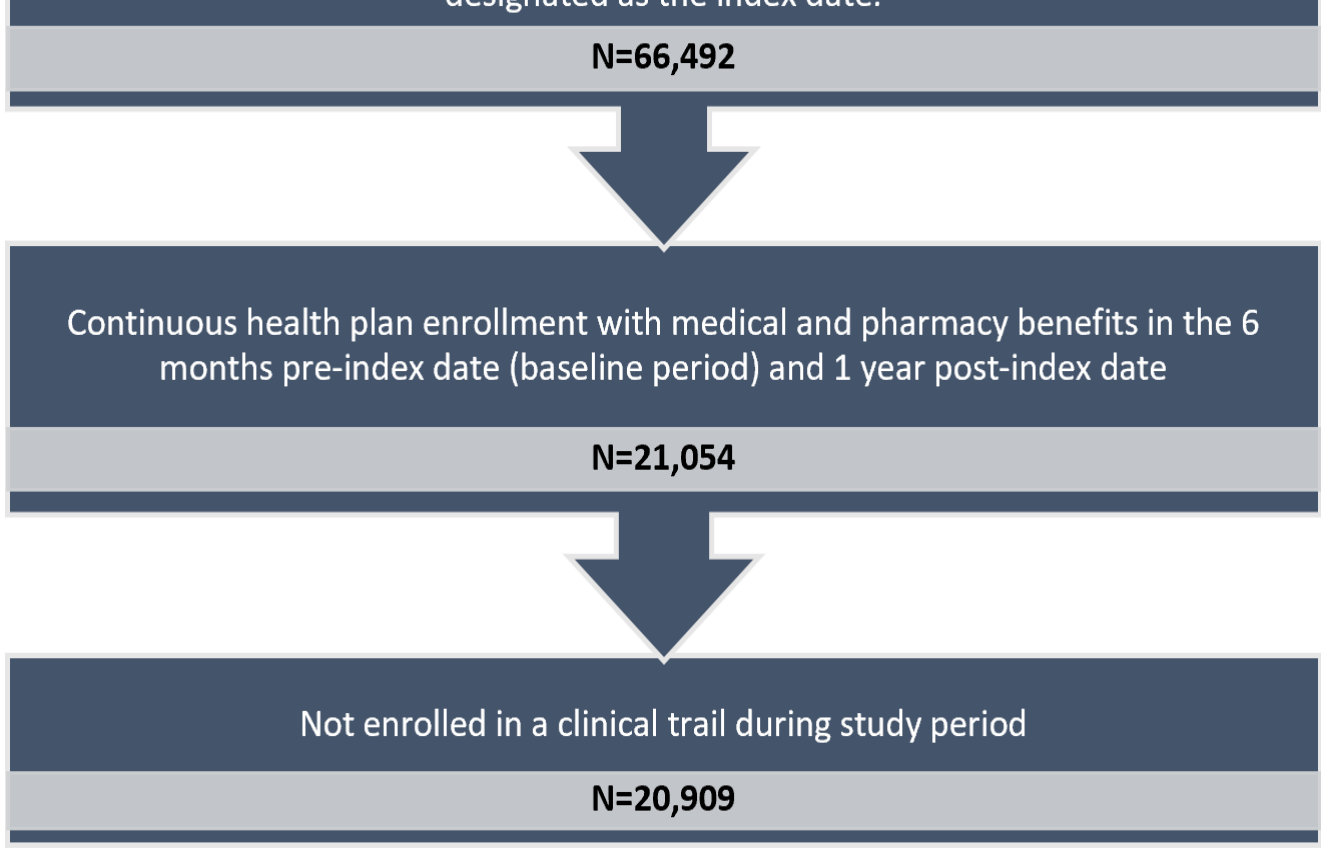


The mean age of the included SCD patients is 17.94 years old (SD=15.17), and $\sim 60 \%(59.25 \%)$ of the enrolled individuals were pediatric patients ( $>18$ years old). The majority $(65.65 \%)$ of the SCD patients were African American and had a CCI score of 0 (75.03\%). Among all the eligible SCD patients, $13 \%(12.82 \%)$ had $\geq 1$ VOC requiring inpatient stay during the half-year baseline period. Pulmonary conditions were the most common cause for baseline comorbidities among the enrolled patients: $13.12 \%$ of them had upper respiratory tract infections, $11.22 \%$ had asthma, and 2.76\% had ACS. Other frequent conditions during the baseline period were infectious and parasitic diseases (18.08\%) and fever (15.51\%). For baseline SCD management, approximately half $(49.03 \%)$ of the patients were prescribed antibiotics, followed by acetaminophen (35.65\%), folic acid (29.19\%), and nonsteroidal anti-inflammatory drugs (NSAIDs) (22.86\%). During the baseline period, $10.89 \%$ of the selected SCD patients had a blood transfusion. For baseline all-cause HRU, 45.50\% of the patients had $\geq 1$ outpatient emergency room visit and $23.88 \%$ had $\geq 1$ inpatient visit with a mean length of stay of 2.90 days $(\mathrm{SD}=9.78)$ (Table 1$)$.

\begin{tabular}{lrcc}
\hline \multicolumn{2}{c}{ Table 1. Baseline Characteristics for Overall SCD Patients } \\
\hline \multirow{2}{*}{ Patient Characteristics - Population A } & & Sickle Cell patients (N=20,909) \\
\cline { 3 - 4 } Age (years) & $<2$ & N/Mean & \%/SD \\
& $2-5$ & 157.94 & 15.17 \\
& $6-11$ & 3662 & $7.55 \%$ \\
& $12-17$ & 3604 & $17.51 \%$ \\
& $18-30$ & 4543 & $17.24 \%$ \\
& $31-45$ & 2521 & $16.94 \%$ \\
& $46-64$ & 1526 & $21.14 \%$ \\
& $65+$ & 53 & $12.06 \%$ \\
& & & $7.30 \%$ \\
\end{tabular}

Sex

Race/Ethnicity

$\begin{array}{rrr}\text { Male } & 9179 & 43.90 \% \\ \text { Female } & 11730 & 56.10 \%\end{array}$

\section{Geographic Region}

$\begin{array}{rcc}\text { White } & 1448 & 6.93 \% \\ \text { Black } & 13727 & 65.65 \% \\ \text { Hispanic } & 2715 & 12.98 \% \\ \text { Other } & 622 & 2.97 \% \\ \text { Unknown } & 2397 & 11.46 \%\end{array}$

\begin{tabular}{lrcc} 
& Northeast & 7671 & $36.69 \%$ \\
& North Central & 2705 & $12.94 \%$ \\
& South & 6715 & $32.12 \%$ \\
Charlson Comorbidity Index Score & West & 3818 & $18.26 \%$ \\
& & 0.42 & 0.96 \\
& 0 & 15688 & $75.03 \%$ \\
& 1 & 3369 & $16.11 \%$ \\
Patients with Baseline VOC & $2-3$ & 1474 & $7.05 \%$ \\
Individual Comorbid Conditions* & $4+$ & 378 & $1.81 \%$ \\
Pulmonary & & 2680 & $12.82 \%$ \\
& & & \\
\hline
\end{tabular}


Table 1. Baseline Characteristics for Overall SCD Patients - Continued

Patient Characteristics - Population A

Sickle Cell patients $(\mathrm{N}=20,909)$

Individual Comorbid Conditions*

Pulmonary

Asthma

Acute Chest Syndrome

Pulmonary embolism

Pulmonary hypertension

Oncologic

Neoplasms benign and malignant

Seizures

Stroke

Spleen

Splenic sequestration

Hypersplenism

Others

$$
\begin{array}{r}
\text { Infectious and parasitic diseases } \\
\text { Fever } \\
\text { Constipation } \\
\text { Iron overload } \\
\text { Aseptic (Avascular) bone necrosis }
\end{array}
$$

Baseline SCD Management

\section{SCD Medication}

Antibiotics
Acetaminophen
Folic Acid
NSAIDs
Opioids (Narcotics)
Hydroxyurea
Iron Chelating therapy

Other SCD Management

$$
\text { Blood transfusions }
$$

Transcranial Doppler ultrasonography

Pneumococcal vaccine

Meningococcal vaccine

Bone marrow transplants

N/Mean

$\% / \mathrm{SD}$

2347

$11.22 \%$

577

$2.76 \%$

111

$0.53 \%$

95

$0.45 \%$

926

$4.43 \%$

647

$3.09 \%$

342

$1.64 \%$

112

$0.54 \%$

20

$0.10 \%$

3781

$18.08 \%$

3242

$15.51 \%$

$5.19 \%$

1085

$2.82 \%$

445

$2.13 \%$

10252

$49.03 \%$

7455

$35.65 \%$

$29.19 \%$

$22.86 \%$

4780

$14.08 \%$

2943

$9.08 \%$

1899

$3.77 \%$

788

2277

$10.89 \%$

1024

$4.90 \%$

1020

$4.88 \%$

226

$1.08 \%$

17

$0.08 \%$

Baseline All-cause Health Care Resource Utilization for all patients (6 months)

$$
\text { Any inpatient stay }
$$

4993

$23.88 \%$

Any outpatient emergency room visit

9513

$45.50 \%$

15342

$73.38 \%$

13692

$65.48 \%$

Any ambulatory surgery center visit

346

$1.65 \%$

5810

$27.79 \%$

7505

$35.89 \%$

17205

\footnotetext{
* Baseline Individual comorbidities $<1 \%$ among overall population were not shown.
} 


\section{Mortality and Complications among Adults and Pediatric SCD Patients}

For adult patients, the follow-up mortality rate was 1.06 in 100 person-years. The percentage of adults who developed VOC requiring an inpatient stay during the first 12 -month follow-up period was 30.86\%; among this percentage of adults with SCD, 74\% had >1 VOC events (Table 3). The rate of VOC events in 100 personyears during the entire follow-up period was 142.20 for adults. The five complications with the highest incidence (in 100 person-years) among adult patients were infectious and parasitic diseases (32.87), fever (15.98), asthma (11.25), ACS (5.71), and aseptic bone necrosis (5.36) (Figure 2).

For pediatric patients, the follow-up mortality rate was 0.14 in 100 person-years. The percentage of children who developed VOC requiring an inpatient stay during the first 12 -month follow-up period was $21.87 \%$; and among this percentage of children with SCD, 59\% had $>1$ VOC events (Table 3). The rate of VOC events was 53.91 for children in 100 person-years. The five complications with the highest incidence among pediatric patients were fever (31.88), infectious and parasitic diseases (27.69), asthma (14.48), ACS (6.98), and gallstones (2.93) (Figure 2).

\section{Figure 2. Incidence of Complications in 100 Person-year for Adult and Pediatric SCD Patients}

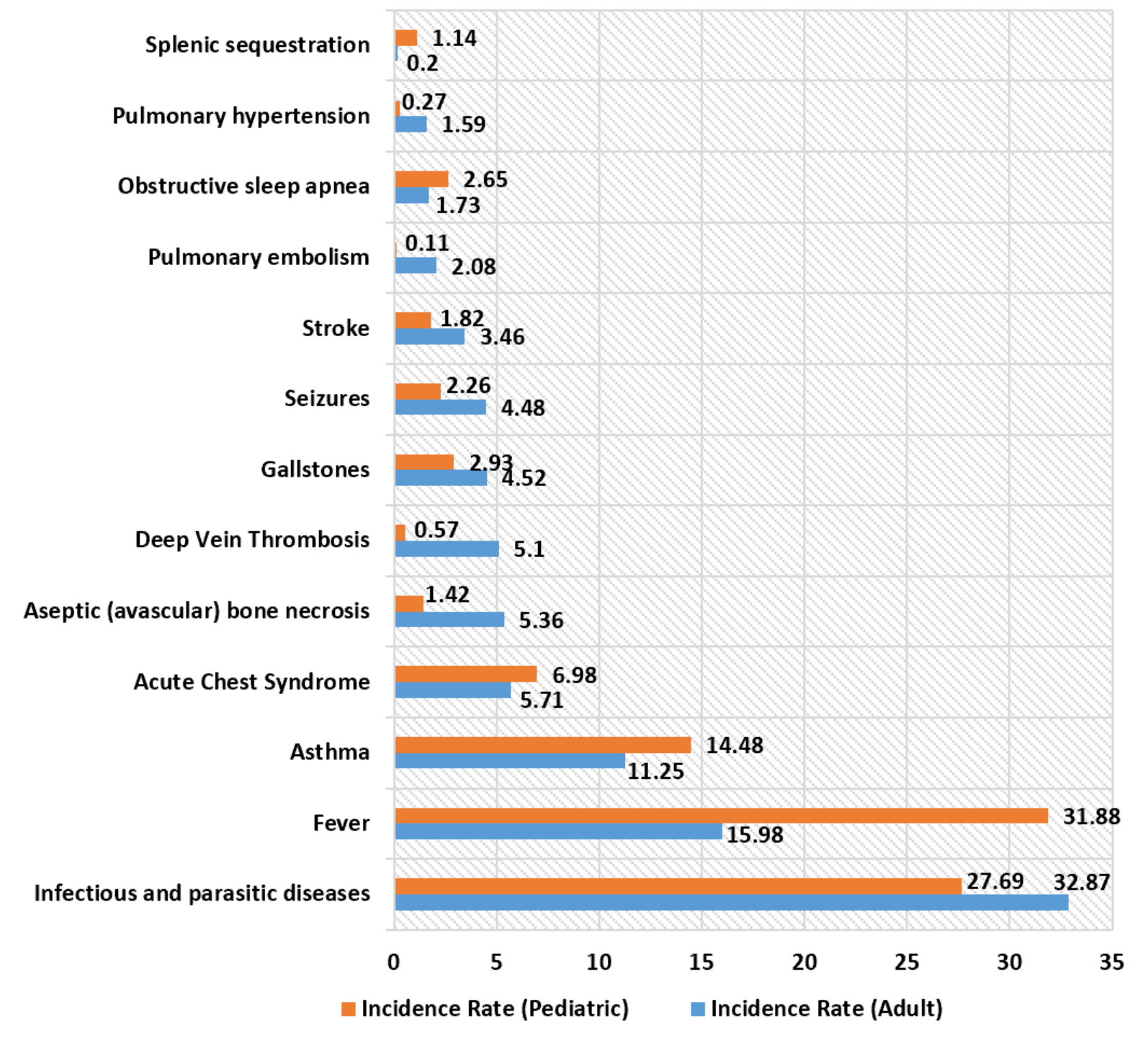




\begin{tabular}{ccccccc}
\hline \multicolumn{6}{c}{ Table 3. Frequency of VOC during } & One-year among Medicaid Population \\
\hline $\begin{array}{c}\text { Frequency } \\
\text { of VOC }\end{array}$ & Among All Patients & Among Adults & A with SCD & Among Children with SCD \\
\hline & $\mathbf{N}$ & Percent & N & $\%$ & N & Percent \\
\hline 0 & 15570 & $74.5 \%$ & 5891 & $69.1 \%$ & 9679 & $78.1 \%$ \\
1 & 1789 & $8.6 \%$ & 677 & $8.0 \%$ & 1112 & $9.0 \%$ \\
2 & 1295 & $6.2 \%$ & 543 & $6.4 \%$ & 752 & $6.1 \%$ \\
3 & 584 & $2.8 \%$ & 286 & $3.4 \%$ & 298 & $2.4 \%$ \\
4 & 466 & $2.2 \%$ & 257 & $3.0 \%$ & 209 & $1.7 \%$ \\
5 & 272 & $1.3 \%$ & 166 & $2.0 \%$ & 106 & $0.9 \%$ \\
6 & 238 & $1.1 \%$ & 150 & $1.8 \%$ & 88 & $0.7 \%$ \\
7 & 127 & $0.6 \%$ & 99 & $1.2 \%$ & 28 & $0.2 \%$ \\
8 & 102 & $0.5 \%$ & 68 & $0.8 \%$ & 34 & $0.3 \%$ \\
9 & 66 & $0.3 \%$ & 49 & $0.6 \%$ & 17 & $0.1 \%$ \\
10 & 73 & $0.4 \%$ & 53 & $0.6 \%$ & 20 & $0.2 \%$ \\
11 & 58 & $0.3 \%$ & 51 & $0.6 \%$ & 7 & $0.1 \%$ \\
12 & 48 & $0.2 \%$ & 40 & $0.5 \%$ & 8 & $0.1 \%$ \\
13 & 38 & $0.2 \%$ & 31 & $0.4 \%$ & 7 & $0.1 \%$ \\
14 & 26 & $0.1 \%$ & 20 & $0.2 \%$ & 6 & $0.1 \%$ \\
15 & 17 & $0.1 \%$ & 15 & $0.2 \%$ & 2 & $0.0 \%$ \\
$>=16$ & 140 & $0.6 \%$ & 125 & $1.4 \%$ & 15 & $0.1 \%$ \\
\hline
\end{tabular}

\section{Relationship between VOC and Life-Threatening Complications for Overall SCD Patients}

Cox models were applied to examine the relationship between the frequency of VOCs and clinical endpoints. After the stepwise model selection, significant patient demographics and baseline clinical characteristics remained in the model. Patients who had a follow-up VOC had a 0.55 higher hazard of death than those without a follow-up VOC (95\% CI [1.19-2.05]; p value=0.0014). Patients with VOC were also more likely to develop life-threatening complications including ACS (HR=58.67; 95\% CI [50.21-68.55]; p value <0.0001), splenic sequestration $(\mathrm{HR}=34.99 ; 95 \%$ CI [30.65-63.13]; $\mathrm{p}$ value $<0.0001)$, pulmonary hypertension $(\mathrm{HR}=4.12$; 95\% CI [3.14-5.41]; p value <0.0001), pulmonary embolism $(\mathrm{HR}=2.82$; 95\% CI [2.21-3.58]; p value $<0.0001)$, and stroke $(\mathrm{HR}=2.26$; 95\% CI [1.94-2.63]; p value $<0.0001)$ (Table 2).

Table 2. Cox Model to Examine the Relationship between VOC and Complications

\begin{tabular}{|c|c|c|c|c|}
\hline \multirow{2}{*}{$\begin{array}{l}\text { Outcomes among Sickle Cell patients } \\
\qquad(\mathrm{N}=20,909)\end{array}$} & \multicolumn{4}{|c|}{ Results from Cox Model for Follow-up VOC } \\
\hline & HR & & & p-value \\
\hline Time-to-Death ${ }^{a}$ & 1.56 & 1.19 & 2.05 & 0.0014 \\
\hline Time-to-Acute Chest Syndrome ${ }^{\mathrm{b}}$ & 58.67 & 50.21 & 68.55 & $<0.0001$ \\
\hline Time-to-Splenic Sequestration ${ }^{c}$ & 43.99 & 30.65 & 63.13 & $<0.0001$ \\
\hline Time-to-Pulmonary Embolism ${ }^{\mathrm{d}}$ & 2.82 & 2.21 & 3.58 & $<0.0001$ \\
\hline Time-to-Stroke & 2.26 & 1.94 & 2.63 & $<0.0001$ \\
\hline Time-to-Pulmonary hypertension ${ }^{f}$ & 4.12 & 3.14 & 5.41 & $<0.0001$ \\
\hline
\end{tabular}

*Significant covariates after the stepwise model selection for each endpoint model include -

a age, sex, race, region, CCI, baseline neoplasms, baseline VOC, baseline use of opioids, NSAIDs, iron chelating therapy, baseline all-cause HRU, follow-up pulmonary embolism, stroke, and pulmonary hypertension; ${ }^{\mathrm{b}}$ age, sex, race, region, baseline use of iron chelating therapy, folic acid, baseline transcranial doppler ultrasonography, baseline all-cause HRU, and follow-up pulmonary embolism; ${ }^{\mathrm{c}}$ age, baseline use of hydroxyurea, and baseline pain crisis; ${ }^{\mathrm{d}}$ age, sex, race, region, CCI, baseline fever, baseline use of opioids, follow-up acute chest syndrome, stroke, and pulmonary hypertension; ${ }^{\mathrm{e}}$ age, sex, race, region, CCI, baseline fever and seizures, baseline use of NSAIDs, iron chelating therapy, tricyclic antidepressants, acetaminophen, baseline blood transfusions and pneumococcal vaccine, baseline VOC, follow-up acute chest syndrome and pulmonary hypertension; ${ }^{\mathrm{f}}$ age, CCI, baseline use of opioids, folic acid, baseline blood transfusion, baseline all-cause HRU, follow-up pulmonary embolism, stroke, and acute chest syndrome 


\section{Discussion}

This was a retrospective claims study using the US Medicaid database that provides real-world insight into clinical outcomes among the SCD population. Specifically, this study examined the mortality and complication rates for pediatric and adult SCD patients and associations between VOCs and major complications. This study provides a better understanding of SCD's clinical burden that may provide insight into VOC management among SCD patients.

The rate of pain crisis events in person-years was 1.42 for adults and 0.54 for children in the Medicaid population, which consists of a higher percentage (43\%) of children. ${ }^{21}$ In Stettler's study using the Optum Normative Health Informatics database with a study period of 2009-2013, the VOC rate among adults diagnosed with SCD was 0.7 in one person-year. ${ }^{22}$ The lower number may be due to Stettler's strict inclusion criteria of VOC that required $\geq 3$ hospitalizations or emergency department visits. Hospitalization rates due to VOC among SCD children and adolescents (0-18 years old) ranged from 0.2 to 1.0 person-years in a large population-based retrospective cohort from 2010 to $2014,{ }^{23}$ which is consistent with our result when estimating an average value of 0.2-1.0 at nearly 0.6. Besides, the number of VOC events captured in our study may be lower than the real number since only VOC episodes in the inpatient setting were identified to reduce noise and ensure that the disease severity for included patients with VOC was on the same level. The feasibility analysis of VOC prevalence from 2009-2013 (detailed results not shown) indicated that when both outpatient and inpatient VOC events were included, the prevalence rates increased from $\sim 30 \%$ to $50 \%$ for all years. Additionally, a large proportion of VOC episodes can be managed at home, as VOC without HRU was reported to be $\sim 23 \%$ among the 31,017 analyzed patient-days. ${ }^{6}$ Therefore, the rate of actual VOC may be underestimated.

Across all complication-related outcomes, pediatric and adult patients were burdened with multiple complications. Some significant complications with high incidence (in 100 person-years) for both children and adults include infectious and parasitic diseases (27.69 and 32.87), fever (31.88 and 15.98), asthma (14.48 and 11.25), and ACS (6.98 and 5.71). ACS is the leading cause of death among SCD patients according to the Cooperative Study of Sickle Cell Disease. It occurs most frequently among children aged 2-4 years (25.3/100 person-years) and decreases gradually to its lowest value in adults (8.8/100 person-years). ${ }^{24}$ In our study, the ACS rates for both adults and children were lower than expected. While this finding may indicate clinical improvements in the management of ACS in the past two decades, further exploration is required to understand this difference.

After applying a stepwise model selection and adjusting demographics, clinical conditions including baseline cancer diagnosis and baseline VOC requiring inpatient care, SCD management including SCD medication use (opioids, NSAIDs, and iron chelating therapy), and HRU, VOC outcomes in our study were-as hypothesizedsignificantly associated with death and a number of SCD-related and life-threatening complications including ACS, stroke, pulmonary hypertension, pulmonary embolism, and splenic sequestration. In Platt's study examining the relationship between VOC and mortality, higher numbers of VOCs were related to higher mortality for those aged $>20$ years but not related to higher mortality for younger patients. ${ }^{25}$ This discrepancy among age groups in Platt's study might be due to the smaller sample size of younger patients, as the author suggests. Therefore, the general mortality trend of this study is likely consistent with Platt's study. The significant relationship between VOC and life-threatening complications is plausible considering the pathological mechanism underlying VOC and all SCD-related complications. Repeated pain episodes can cause bone marrow infarction and necrosis, which lead to pulmonary fat embolism, ACS, and other cardiovascular complications such as stroke and pulmonary hypertension. ${ }^{26}$ Though the exact pathophysiology of how SCD VOC may contribute to further crisis events is not completely clear, previous studies support the evidence that severe pain symptoms often precede such acute events and may be an indicator for special attention for treatment and prevention of further severe complications. ${ }^{27}$ 
There were also several limitations to this study. While claims data are extremely valuable for the efficient and effective examination of health care outcomes, claims data are collected for payment and not research. First, since VOC events were identified only by using inpatient diagnosis claims, the sample size of patients with VOC might be underestimated since many crisis events can be managed at home; this might reduce the number of VOC and diminish the associations between VOC and complications. In addition, the presence of a diagnosis code on a medical claim cannot confirm the presence of disease, as the diagnosis code may be incorrectly coded or included as rule-out criteria rather than actual disease, which would impact the inclusion of SCD and VOC patients. Second, this study might not be generalizable to other populations. The Medicaid population consists of nearly half of children aged $<18$ years. This study setting is within the Medicaid population that consists of people with disabilities, low-income children below a certain wage, pregnant women, parents of Medicaideligible children who meet certain income requirements, and low-income seniors. ${ }^{28}$ These populations are more likely to have unmet needs in health care resource services. In this study, we excluded individuals with dual eligibility for both Medicaid and Medicare since data of these observations are not complete: due to limited availability, the data of managed care plan patients only include 14 states, and the study period until December 2013 was the most recent data at the time of study. With a mean follow-up time of 2.7 years for all included patients, the mortality rates in our study might not be generalized to long-term mortality and should only be compared to the population with a similar follow-up length.

SCD patients have a substantial burden of complications. Patients experiencing painful crises requiring inpatient care had a significantly higher risk of experiencing subsequent crisis and other life-threatening SCD-related complications. We found strong evidence that VOC is a key risk factor for severe clinical outcomes. Policymakers and physicians should consider how to prevent severe clinical conditions by improving health care resources and access for those patients with VOCs.

\section{Authorship Contributions}

NS contributed to the interpretation of the data, wrote the manuscript and substantially contributed to critical revisions of the intellectual content.

$\mathrm{MB}, \mathrm{RH}, \mathrm{SA}$, and JP conceptualized and designed the study, contributed to the interpretation of the data, and substantially contributed to critical revisions of the intellectual content.

LX and HY conceptualized and designed the study, contributed to the acquisition and interpretation of the data, and substantially contributed to critical revisions of the intellectual content.

\section{Conflict of Interest Disclosures}

NS is a consultant and speaker for Novartis. MB, RH, SA, and JP are paid employees of Novartis Pharmaceuticals, the study sponsor. LX is a paid employee of STATinMED Research, a paid consultant to Novartis Pharmaceuticals. HY declares no conflict of interest. 


\section{References}

1 Yawn BP, Buchanan GR, Afenyi-Annan AN, et al. Management of sickle cell disease: summary of the 2014 evidence-based report by expert panel members. JAMA. 2014;312(10):1033-1048.

${ }^{2}$ Centers for Disease Control and Prevention. What is Sickle Cell Disease? https://www.cdc.gov/ncbddd/ sicklecell/facts.html. Accessed 16 March 2017.

3 American Society of Hematology. Sickle Cell Trait. www.hematology.org/Patients/Anemia/Sickle-Cell-Trait. aspx. Accessed 16 March 2017.

${ }^{4}$ Centers for Disease Control and Prevention. Sickle Cell Disease (SCD) Data \& Statistics. https://www.cdc. gov/ncbddd/sicklecell/data.html. Accessed 16 March 2017.

5 Yale SH, Nagib N, Guthrie T. Approach to the vaso-occlusive crisis in adults with sickle cell disease. Am Fam Physician. 2000;61(5):1349-1356.

${ }^{6}$ Smith WR, Penberthy LT, Bovbjerg VE, et al. Daily assessment of pain in adults with sickle cell disease. Ann Intern Med. 2008;148(2):94-101.

7 Dampier C, Ely E, Brodecki D, O’Neal P. Home management of pain in sickle cell disease: a daily diary study in children and adolescents. J Pediatr Hematol Oncol. 2002;24(8):643-647.

${ }^{8}$ Dampier C, Ely B, Brodecki D, O’Neal P. Characteristics of pain managed at home in children and adolescents with sickle cell disease by using diary self-reports. J Pain. 2002;3(6):461-470.

9 Centers for Disease Control and Prevention. Sickle Cell Disease (SCD) Complications \& Treatment. https:// www.cdc.gov/ncbddd/sicklecell/treatments.html. Accessed 23 August 2017.

${ }^{10}$ Smith WR, Scherer M. Sickle-cell pain: advances in epidemiology and etiology. Hematology Am Soc Hematol Educ Program. 2010;2010:409-415.

${ }^{11}$ Novelli EM, Gladwin MT. Crises in sickle cell disease. Chest. 2016;149(4):1082-1093.

${ }^{12}$ Gill FM, Sleeper LA, Weiner SJ, et al. Clinical events in the first decade in a cohort of infants with sickle cell disease. Cooperative Study of Sickle Cell Disease. Blood. 1995;86(2):776-783.

${ }^{13}$ Lanzkron S, Carroll CP, Haywood C Jr. Mortality rates and age at death from sickle cell disease: U.S., 19792005. Public Health Rep. 2013;128(2):110-116.

${ }^{14}$ Gladwin MT, Sachdev V, Jison ML, et al. Pulmonary hypertension as a risk factor for death in patients with sickle cell disease. N Engl J Med. 2004;350(9):886-895.

${ }^{15}$ Fitzhugh CD, Lauder N, Jonassaint JC, et al. Cardiopulmonary complications leading to premature deaths in adult patients with sickle cell disease. Am J Hematol. 2010;85(1):36-40.

${ }^{16}$ Vichinsky EP, Neumayr LD, Earles AN, et al. Causes and outcomes of the acute chest syndrome in sickle cell disease. N Engl J Med. 2000;342(25):1855-1865.

${ }^{17}$ Darbari DS, Wang Z, Kwak M, et al. Severe painful vaso-occlusive crises and mortality in a contemporary adult sickle cell anemia cohort study. PloS One. 2013;8(11):e79923.

${ }^{18}$ Kanter J, Kruse-Jarres R. Management of sickle cell disease from childhood through adulthood. Blood Rev. 2013;27(6):279-287.

${ }^{19}$ Sobota AE, Umeh E, Mack JW. Young adult perspectives on a successful transition from pediatric to adult care in sickle cell disease. J Hematol Res. 2015;2(1):17-24.

${ }^{20}$ Charlson ME, Charlson RE, Peterson JC, Marinopoulos SS, Briggs WM, Hollenberg JP. The Charlson comorbidity index is adapted to predict costs of chronic disease in primary care patients. J Clin Epidemiol. 2008;61(12):1234-1240. 
${ }^{21}$ The Kaiser Family Foundation. State Health Facts: FY2014 Medicaid Enrollees by Enrollment Group. https://www.kff.org/medicaid/state-indicator/distribution-of-medicaid-enrollees-by-enrollment-group/? currentTimeframe $=0 \&$ sortModel $=\% 7 \mathrm{~B} \% 22$ colld $\% 22: \% 22$ Location $\% 22, \% 22$ sort $\% 22: \% 22$ asc $\% 22 \% 7 \mathrm{D}$. Accessed December 19, 2018.

${ }^{22}$ Stettler N, McKiernan CM, Melin CQ, Adejoro OO, Walczak NB. Proportion of adults with sickle cell anemia and pain crises receiving hydroxyurea. JAMA. 2015;313(16):1671-1672.

${ }^{23}$ Theodore R, Barry V, Quarmyne MO, DampierC, Lane PA. Inpatient utilization among children and adolescents with sickle cell disease: analysis of a large populated-based retrospective cohort. Blood. 2015;126(23):3262.

${ }^{24}$ Castro O, Brambilla DJ, Thorington B, et al. The acute chest syndrome in sickle cell disease: incidence and risk factors. The Cooperative Study of Sickle Cell Disease. Blood. 1994;84(2):643-649.

${ }^{25}$ Platt OS, Thorington BD, Brambilla DJ, et al. Pain in sickle cell disease. rates and risk factors. N Engl J Med. 1991;325(1):11-16.

${ }^{26}$ Herman M, Chaudhry S. Wong E (ed). Sickle Cell Disease (SCD). http://www.pathophys.org/scd/. Accessed 15 February 2018.

${ }^{27}$ Al-Salem AH. Splenic complications of sickle cell anemia and the role of splenectomy. ISRN Hematol. 2011;2011:864257.

${ }^{28}$ Krieger JW, Connell FA, LoGerfo JP. Medicaid prenatal care: a comparison of use and outcomes in fee-forservice and managed care. Am J Public Health. 1992;82(2):185-190. 